\title{
Effect of dynamic strain rate on micro-indentation properties of pure aluminum
}

\author{
Hiroyuki Yamada ${ }^{1, \text { a }}$, Midori Hotta ${ }^{2}$, Tsuyoshi Kami ${ }^{3}$, Nagahisa Ogasawara ${ }^{1}$, and Xi Chen ${ }^{4}$ \\ ${ }^{1}$ National Defense Academy, School of Systems Engineering, 1-10-20 Hashirimizu Yokosuka Kanagawa, Japan \\ ${ }^{2}$ National Defense Academy, Graduate Student, School of Systems Engineering, 1-10-20 Hashirimizu Yokosuka \\ Kanagawa, Japan \\ ${ }^{3}$ Columbia University, Graduate Student, 500 West 120th Street, New York, USA \\ ${ }^{4}$ Columbia University, 500 West 120th Street, New York, USA
}

\begin{abstract}
Indentation is widely used to investigate the elastic and plastic properties of mechanical materials, which includes the strain rate sensitivity. The indentation exhibits an inhomogeneous strain distribution in contrast to compression and tensile tests with homogeneous deformation. Thus, the strain rate of the indentation may form the inhomogeneous distribution. Therefore, the effect of strain rate distribution of the indentation on pure aluminum with respect to the strain rate dependence of strength in order to clarify the effect of the strain rate on the indentation technique. First, the numerical simulation was established using the Cowper-Symonds equation as the dynamic constitutive equation. Secondary, the strain rate distribution was calculated from the equivalent plastic strain distribution. The strain rate distribution was quite different from the strain distribution, which showed that the strain rate at the crater rim was higher than that beneath the indenter. Finally, we try to perform the averaging of strain rate distribution in order to make an index of strain rate in the indentation. The average of strain rate distribution was calculated using the equivalent plastic strain above a boundary value that is the critical strain and the representative strain. There is correlation between the average strain rate and the loading curvature, which shows that the average strain rate can express as the representative of strain rate for the indentation technique.
\end{abstract}

\section{Introduction}

Instrumented indentation tests are widely used to investigate the elastic and plastic properties of mechanical materials [1-3]. A rigid indenter penetrates normally into a homogeneous solid, where the indentation load $(P)$ and displacement $(h)$ are continuously recorded during loading and unloading.

In the micro-indentation test, the hardness has been reported to increase with decreasing indentation depth $[4,5]$. Doerner and Nix [4] pointed out that the material strength was affected by the strain rate in the indentation test. When the indentation test is performed at a constant displacement rate, $\dot{h}$, the strain rate can be defined as follows:

$$
\dot{\varepsilon}_{i}=c(\dot{h} / h) .
$$

where $c$ is the constant and $\dot{\varepsilon}_{i}$ is called indentation strain rate. Thus, the Eq. (1) indicates that the strain rate becomes very high as the indentation depth decreases.

While the pure aluminum with strain rate dependence of strength has been evaluated for investigating the effect of strain rate. In a previous study, the authors [3] have investigated properties of 99.999 mass\% pure aluminum (hereinafter referred to as $5 \mathrm{~N}$ aluminum). Figure 1 shows the typical true stress-strain curves in the quasi-static test and the impact test for $5 \mathrm{~N}$ aluminum. It was confirmed that the flow stress was increased with increasing the strain

\footnotetext{
${ }^{a}$ Corresponding author: ymda@nda.ac.jp
}

rate. In addition, the typical load-displacement curves for $5 \mathrm{~N}$ aluminum in the indentation test are shown in Fig. 2. During the loading process, the load increased with increasing loading rate. Thus, it was revealed that the load of the micro-indentation test was affected by the loading rate. As shown in Figs. 1 and 2, the effect of loading rate on load during indentation was in good agreement with the trend of the strain rate dependence of flow stress.

In addition, the authors [6] have also investigated the effect of high strain rate on the load-displacement relation by comparing the results of a micro-indentation test at a loading rate of $70 \mathrm{mN} / \mathrm{s}$ and the results obtained by the finite element method (FEM) analysis using the annealed pure copper having strain rate dependence of strength. As a result of the FEM analysis, the equivalent plastic strain rate at the early stage of testing reached the dynamic strain rate, approximately $10^{1} \mathrm{~s}^{-1}$, underneath the sharp indenter in the micro-indentation test. This result has shown the effect of strain rate on the indentation.

However, the indentation exhibits an inhomogeneous strain distribution in contrast to the compression and the tensile tests with homogeneous deformation. Thus, the strain rate of the indentation may form the inhomogeneous distribution.

The purpose of this study is to investigate the effect of strain rate distribution of the indentation on pure aluminum with respect to strain rate dependence of strength in order to clarify in detail the effect of high strain rate on the indentation test.

This is an Open Access article distributed under the terms of the Creative Commons Attribution License 4.0, which permits unrestricted use, distribution, and reproduction in any medium, provided the original work is properly cited. 


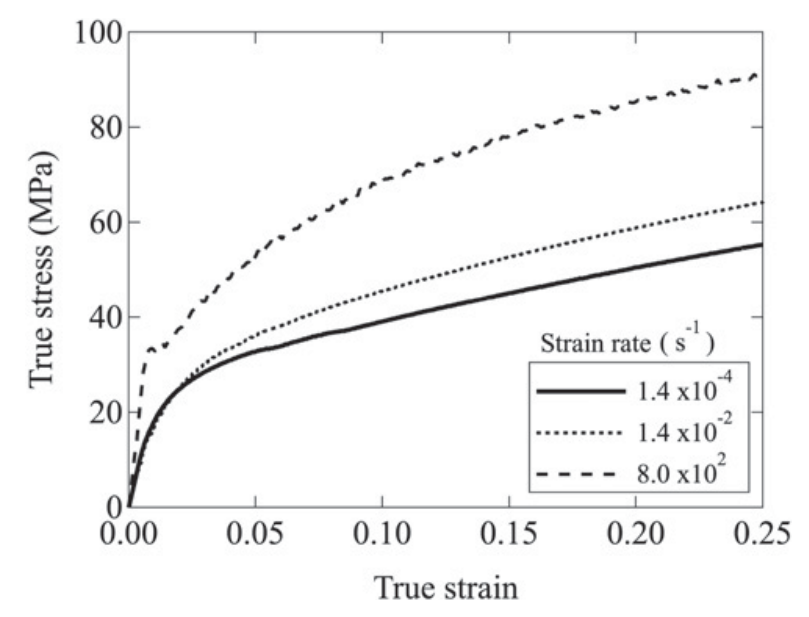

Figure 1. True stress-strain curves for $5 \mathrm{~N}$ aluminum in the quasistatic test and the impact test.

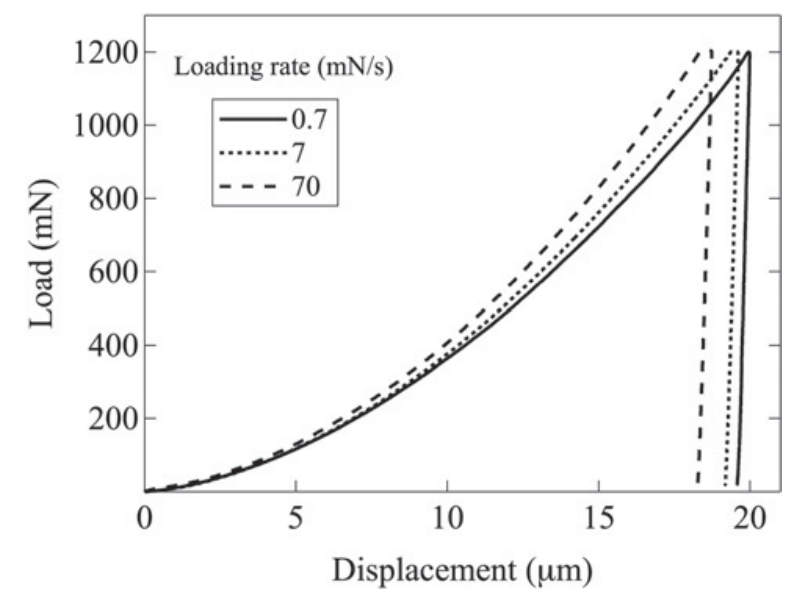

Figure 2. Load-displacement curves for $5 \mathrm{~N}$ aluminum in the indentation test.

\section{The effect of the indentation strain rate}

The flow stress with respect to the strain rate could be expressed with exponential function defined by following equations.

$$
\sigma=\sigma_{0}\left[1+\left(\frac{\dot{\varepsilon}}{\gamma}\right)^{m}\right]
$$

where $\sigma_{0}$ is the static stress, $m$ and $\gamma$ are the strain rate parameters. The above equation is called the CowperSymonds constitutive equation. This constitutive equation is widely used to represent the strain rate sensitivity of metal materials in a simple way.

In general, the FEM analysis has the implicit and the explicit methods. The latter, such as LS-DYNA, is designed for dynamic analysis, which means the problem of the stress wave propagation. In a previous study [6], the authors used the explicit methods in order to use the Cowper-Symonds model, because the high strain rate phenomenon was confirmed in the indentation test. However, the typical indentation test using commercially apparatus has longer test duration than the high strain rate test to evaluate the dynamic and the impact phenomena. Therefore, the mass scaling technique has been used in

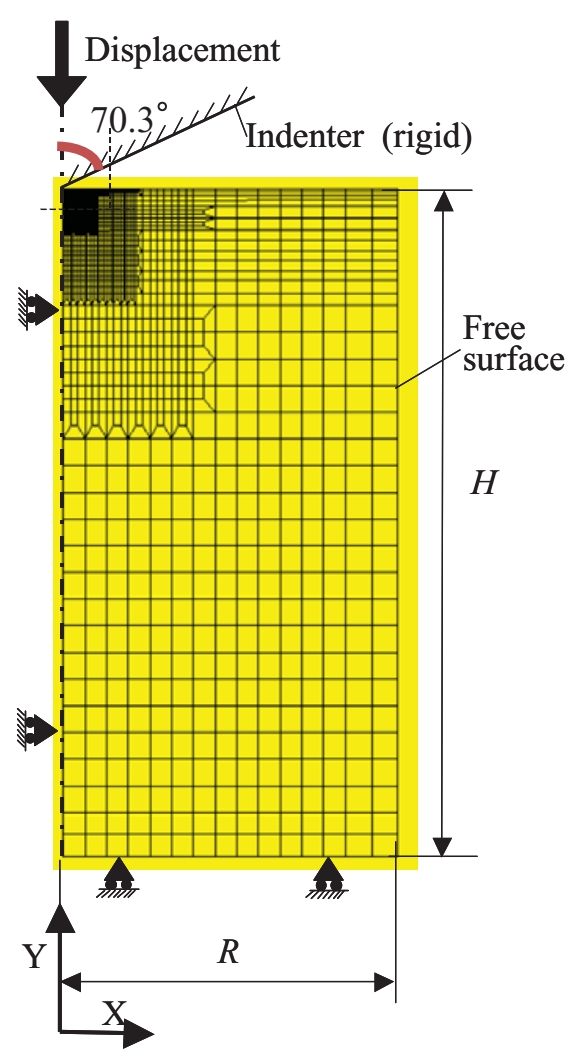

Figure 3. Simulation model.

the previous study [6], which can shorten the analysis time using the explicit method. Thereby, it is necessary to make the density of indenter 10000 times to shorten the analysis time. However, this leads to the inertia force in specimen due to the acceleration. The actual indenter was moved at a few $\mu \mathrm{m} / \mathrm{s}$, which did not take place the inertia force in specimen. Thus, we try to use the implicit code for the analysis of the indentation affected by the strain rate.

In this study, the numerical simulations were performed using the commercial finite element package ANSYS, the implicit code. Figure 3 shows the simulation model. For simplicity, the indentation simulation was modeled as an axisymmetric and elasto-plastic analysis. The conical indenter with included with half apex angle of $70.3^{\circ}$ was modeled as a rigid body. This gives approximately the same ratio of indentation depth to contact area as the Berkovich indenter. A typical mesh for the axisymmetric indentation model comprises about 4-node elements. The mesh has approximately 29,600 elements. As shown in Fig. 3, the mesh of the model is designed to become coarser with distance from the indenter tip in order to reproduce the strain distribution during the indentation. The minimum element size was $0.075 \mu \mathrm{m} \times 0.125 \mu \mathrm{m}$ underneath the indenter. Thus, more accurate results and convergences can be obtained.

In the indentation test, there are two indenter control methods: the load control (loading rate, $\dot{P}$, is constant) and the displacement control (displacement rate, $\dot{h}$, is constant). When used the load control, the strain rate changes depending on materials during indentation. Therefore, the displacement control method is used in this study. To evaluate the role of indenter velocity, the analysis 


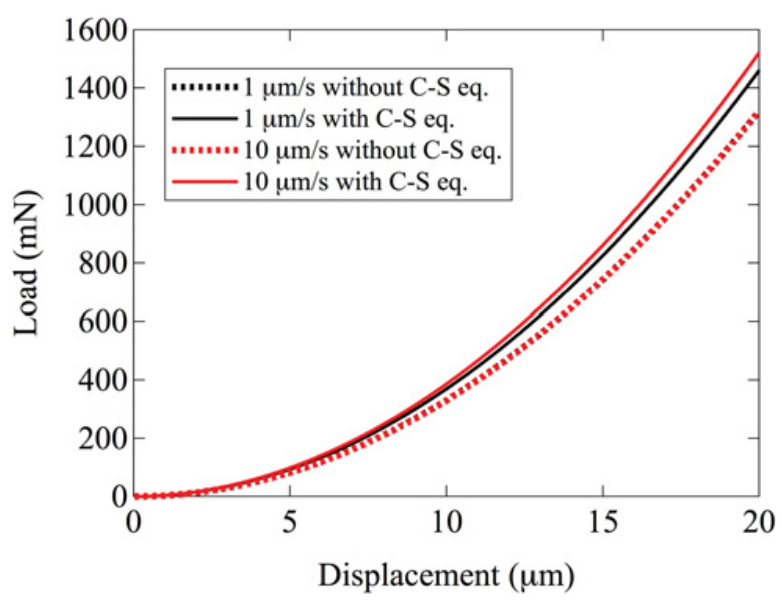

Figure 4. Load-displacement curves obtained through the FEM analysis at displacement rate of $1 \mu \mathrm{m} / \mathrm{s}$ and $10 \mu \mathrm{m} / \mathrm{s}$.

was performed at $\dot{h}=1$ and $10 \mu \mathrm{m} / \mathrm{s}$. The friction between indenter and specimen surface is 0.15 , which assumes the friction between diamond and metal. We chose to the highpure aluminum with 99.999 mass \% purity for the material model, which is proved to have the strain rate dependence as shown in Fig. 1. The values of strain rate parameters of the Cowper-Symonds model are $m=0.16$ and $\gamma=5000$.

\section{The effect of strain rate distribution on indentation}

\subsection{Loading curvature-displacement relationship}

Under quasi-static conditions, the loading segment of the load-displacement relation obeys a parabolic relation, known as Kick's law:

$$
P=C h^{2}
$$

where $C$ is the loading curvature, which is a constant value. The loading curvature depends on both the elastoplastic properties of materials and the indenter angle. It was known [4] that the loading curvature decreased with increasing displacement immediately after indentation, and then this value was convergent, regardless of the loading rate. This is because the indentation was affected by the strain rate at the early stage of testing.

The load-displacement curves were obtained through the FEM analysis at $\dot{h}=1 \mu \mathrm{m} / \mathrm{s}$ and $10 \mu \mathrm{m} / \mathrm{s}$ (Fig. 4). And then the loading curvature-displacement relations obtained through the load-displacement curves were calculated using Eq. (3), as shown in Fig. 5. In the FEM analysis without the Cowper-Symonds model, the loading curvature kept constant, and the load-displacement curves at $\dot{h}=1 \mu \mathrm{m} / \mathrm{s}$ and $10 \mu \mathrm{m} / \mathrm{s}$ coincided. On the other hand, the decrease of the loading curvature immediately after initial indentation and the convergence were observed when used the Cowper-Symonds model, regardless of the $\dot{h}$. In addition, the loading curvature was increased when the displacement rate was increased. Thus, we can replicate the effect of strain rate on the indentation during the loading using the implicit method, as is the case in the explicit method [6].

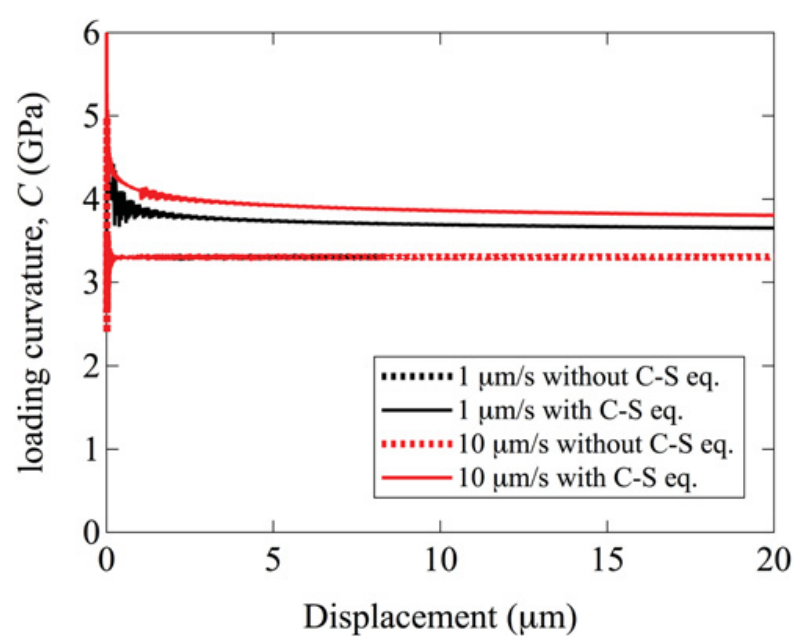

Figure 5. Loading curvature displacement curves obtained from Fig. 4 at displacement rate of $1 \mu \mathrm{m} / \mathrm{s}$ and $10 \mu \mathrm{m} / \mathrm{s}$.

\subsection{Relationship between strain and strain rate distribution}

Figure 6 shows the equivalent plastic strain distributions at $h=0.2,2,20 \mu \mathrm{m}$ and $\dot{h}=1 \mu \mathrm{m} / \mathrm{s}$. It was clear that the strain was concentrated beneath the indenter. Furthermore, the relationship between the equivalent plastic strain distribution and the displacement indicated the correlation.

In this study, we cannot obtain directly the strain rate distribution using ANSYS software. Thus, the strain rate at each node point was calculated from the equivalent plastic strain, $\varepsilon_{\text {plastic }}$, as follows:

$$
\dot{\varepsilon}_{\text {node }}=\frac{d \varepsilon_{\text {plastic }}}{d t}
$$

where, $d t$ is the output time step of the simulation, which is $2 \mathrm{~ms}$ in this study. The strain rate distribution was shown using the numerical software, MATLAB. Figure 7 shows the strain rate distributions at $h=0.2,2,20 \mu \mathrm{m}$ and $\dot{h}=1 \mu \mathrm{m} / \mathrm{s}$. It was revealed that the value for the strain rate distribution is decreased with increasing the displacement.

The strain rate distribution was quite different from the strain distribution at each displacement. As shown in Fig. 6, the strain beneath the indenter keeps high when the displacement is increased, which indicate that there is little change in the amount of strain around the indenter tip. On the other hand, it was confirmed that the strain rate at the crater rim was higher than that beneath the indenter. The strain of the crater rim changes newly with increasing the displacement, which means that the amount of the strain rate change was high. We infer that the high strain rate area does not express as a representative of strain rate for the indentation, since that area is very small in comparison with overall strain rate distribution.

The strain rate distributions for the same indentation strain rate calculated from Eq. (1) were compared. Figure 8 shows the strain rate distribution at $\dot{h}=10 \mu \mathrm{m} / \mathrm{s}$ and $h=2 \mu \mathrm{m}$, which is the condition of $\dot{\varepsilon}_{i}=5 \mathrm{c}$. The value of the strain rate distribution at $\dot{h}=1 \mu \mathrm{m} / \mathrm{s}$ and $h=0.2 \mu \mathrm{m}$ almost coincided with that at $\dot{h}=10 \mu \mathrm{m} / \mathrm{s}$ 


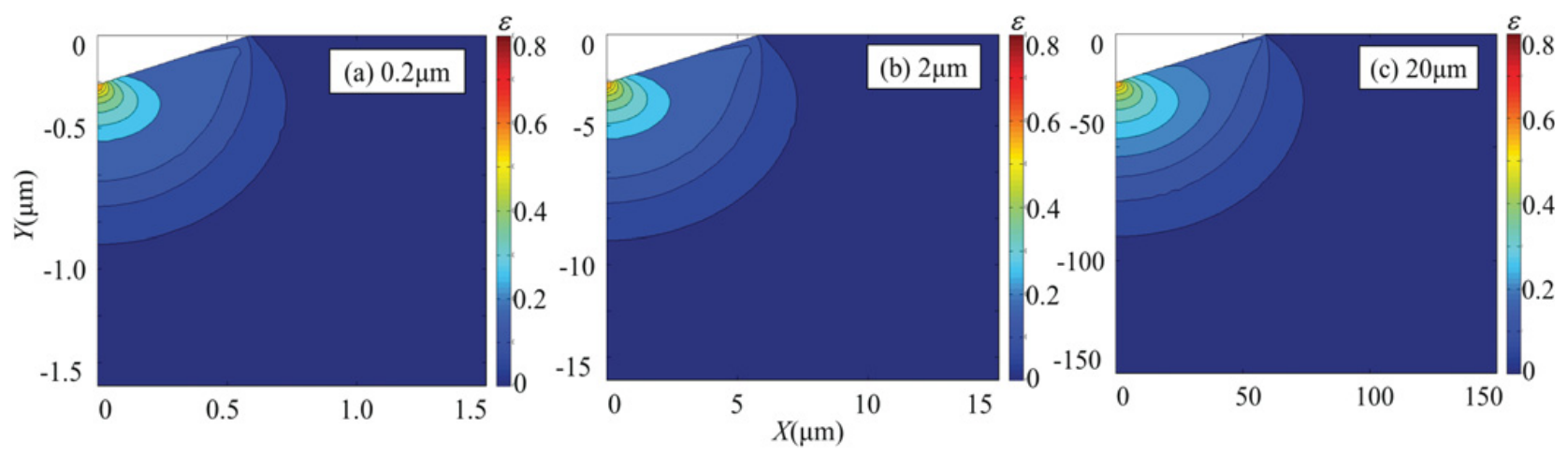

Figure 6. Equivalent plastic strain distributions at displacement rate of $1 \mu \mathrm{m} / \mathrm{s}$.
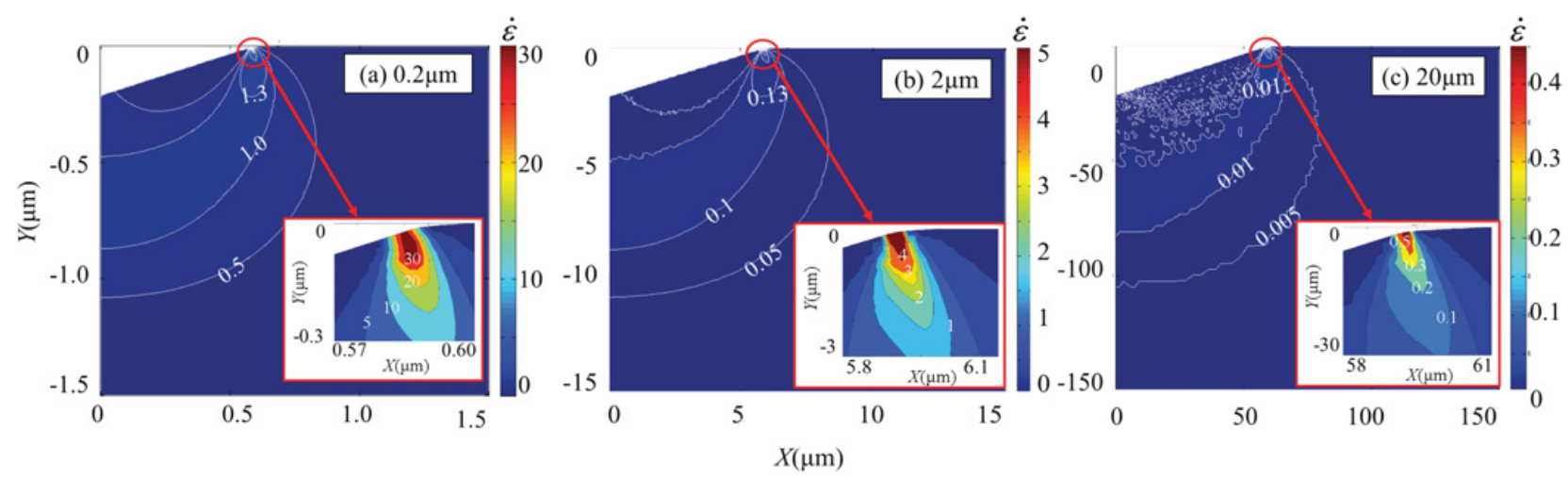

Figure 7. Strain rate distributions at displacement rate of $1 \mu \mathrm{m} / \mathrm{s}$.

and $h=2 \mu \mathrm{m}$. This result shows that these distributions of the same indentation strain rate have the similarity rule.

\section{Introduction of average strain rate}

\subsection{Definition of average strain rate}

As discussed in the previous chapter, the effect of the strain rate distribution on indentation is not apparent in the results of FEM analysis. Therefore, we try to perform the averaging of strain rate distribution in order to make an index of strain rate in the indentation. In this study, the average of strain rate distribution is calculated using the equivalent plastic strain above a boundary value, which defines as average strain rate, $\dot{\varepsilon}_{a v}$. The candidates for this boundary value are the critical strain, $\varepsilon_{c}$, and the representative strain, $\varepsilon_{R}$.

Liu et al. [7] pointed out that the critical strain was identified as the upper boundary of the detectable range of indentation deformation, and moderate tailoring of the constitutive behavior beyond this range cannot be effectively detected by the reverse analysis of the $P-h$ curve. The authors [8] proposed that the critical strain as follows:

$$
\varepsilon_{c}=\left(2.3 n^{2}-0.3 n-1.0\right)(\alpha-88.4) .
$$

where $n$ is the work-hardening exponent and $\alpha$ is a cone with half apex angle. When using the conical indenter with half apex angle of $70.3^{\circ}, \varepsilon_{c}$ is 0.15 .

Dao et al. [9] introduced the concept of the representative strain, which is used for the indentation

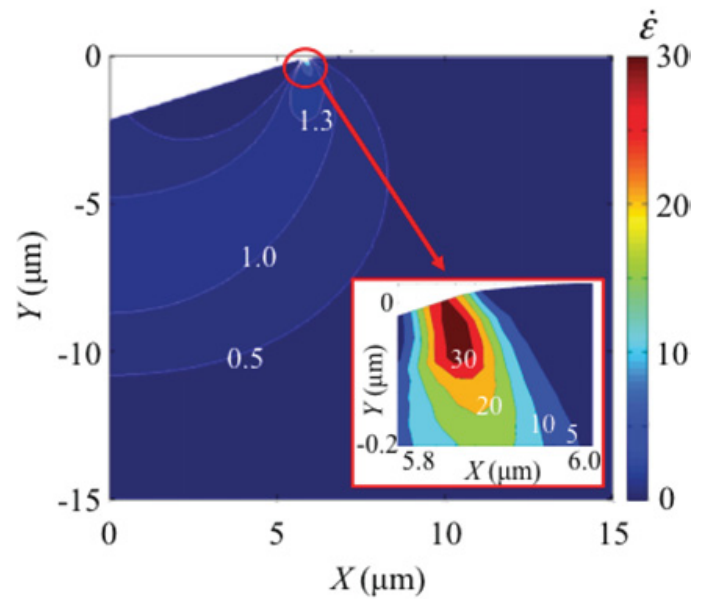

Figure 8. Strain rate distribution under the same indentation strain rate with Fig. 7 (a) $(h=10 \mu \mathrm{m} / \mathrm{s}, h=2 \mu \mathrm{m})$.

analysis to normalize the load-displacement curves of the indentation. The authors [10] proposed more exact the representative strain as follows:

$$
\varepsilon_{R}=0.0638 \cot \alpha
$$

In the present study, $\varepsilon_{R}$ is 0.03 .

\subsection{Calculation of average strain rate}

A process of calculation for the average strain rate is shown below. 


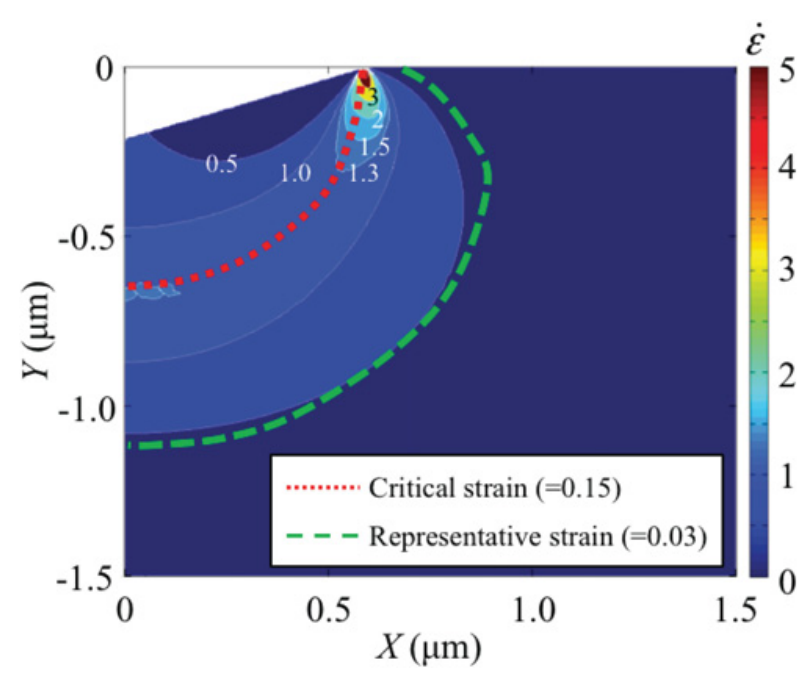

Figure 9. Relationship between the boundary value and the strain rate distribution at $\dot{h}=1 \mu \mathrm{m} / \mathrm{s}$ and $h=0.2 \mu \mathrm{m}$, which shows the miniature of the strain rate range of Fig. 7(a).

(a) The strain rate of each element, $\dot{\varepsilon}_{\text {elm }}$, is calculated as follows:

$$
\dot{\varepsilon}_{\text {elm }}=\frac{1}{4} \sum_{k=1}^{4} \dot{\varepsilon}_{k}
$$

where, $\dot{\varepsilon}_{k}$ is the strain rate at each node point making up the element.

(b) An area filling $\varepsilon_{e l m} \geq \varepsilon_{c}$ or $\varepsilon_{R}$ is extracted in the equivalent plastic strain distributions.

(c) A volume size at each element in the area noted above, $V_{\text {elm }}$, is calculated, since the elements are different from each other in this study. The area at each element, $S_{\text {elm }}$, is calculated using the shoelace formula as follows:

$$
S_{\text {elm }}=\frac{1}{2} \sum_{k=1}^{4}\left(x_{k} y_{k+1}-x_{k+1} y_{k}\right)
$$

where $x_{k}$ and $y_{k}$ are coordinate data of each node making up the element. $V_{\text {elm }}$ is calculated using Pappus-Guldinus theorem as follows:

$$
V_{e l m}=S_{e l m} \times 2 \pi \times \frac{1}{4} \sum_{k=1}^{4} x_{k} .
$$

(d) The average strain rate is calculated as follows:

$$
\dot{\varepsilon}_{a v, X}=\frac{\sum\left(\dot{\varepsilon}_{e l m} \times V_{e l m}\right)}{\sum V_{e l m}}
$$

where, $\dot{\varepsilon}_{a v, X}$ is the average strain rate at each boundary value.

\subsection{Relationship between average strain rate and loading curvature}

Figure 9 shows the relationships between the boundary value and the strain rate distribution at $\dot{h}=1 \mu \mathrm{m} / \mathrm{s}$ and $h=0.2 \mu \mathrm{m}$. The representative strain encompassed a broad strain rate range area compared to the critical strain.
Table 1. The relationship between $\dot{\varepsilon}_{a v, R}$ and the loading curvature.

\begin{tabular}{|c|c|c|c|c|}
\hline \multirow{2}{*}{ Data } & \multicolumn{2}{|c|}{$\dot{\varepsilon}_{a v, R}\left(\mathrm{~s}^{-1}\right)$} & \multicolumn{2}{c|}{$C(\mathrm{GPa})$} \\
\cline { 2 - 5 } & $1 \mu \mathrm{m} / \mathrm{s}$ & $10 \mu \mathrm{m} / \mathrm{s}$ & $1 \mu \mathrm{m} / \mathrm{s}$ & $10 \mu \mathrm{m} / \mathrm{s}$ \\
\hline 0.2 & $7.90 \times 10^{-1}$ & $7.63 \times 10^{0}$ & 4.03 & 4.33 \\
\hline 2 & $7.86 \times 10^{-2}$ & $7.88 \times 10^{-1}$ & 3.80 & 4.01 \\
\hline 20 & $6.76 \times 10^{-3}$ & $7.95 \times 10^{-2}$ & 3.65 & 3.81 \\
\hline
\end{tabular}

When calculated using the equation (10) at $\dot{h}=1 \mu \mathrm{m} / \mathrm{s}$ and $h=0.2 \mu \mathrm{m}$, the average strain rates of the critical strain and the representative strain were $7.25 \times 10^{-1} \mathrm{~s}^{-1}$ and $7.90 \times 10^{-1} \mathrm{~s}^{-1}$, respectively. In both the boundary strains, the average strain rates reached approximately $10^{-1} \mathrm{~s}^{-1}$. In this condition, the highest strain rate is approximately $3 \times 10^{1} \mathrm{~s}^{-1}$, as shown in Fig. 7(a). It was thus clear that the average strain rate was not affected by the highest strain rate around the crater rim.

The relationship between $\dot{\varepsilon}_{a v, X}$ and the loading curvature were investigated. As an example, the terms of $\dot{\varepsilon}_{a v, R}$ is listed in Table 1 . The average strain rate was decreased with increasing the displacement. This is because the increase in volume at low strain rate area becomes marked when the displacement was increased. In addition, it was clarified that the loading curvature was decreased with increasing the displacement, which indicates the similar trend of the value of $\dot{\varepsilon}_{a v, R}$.

Therefore, the average strain rates and the loading curvature under the same indentation strain rates were compared. The same indentation strain rates are indicated by the arrows in Table 1 . The average strain rate and the loading curvature for the same indentation strain rates showed almost the same values. Thus, there is correlation between the average strain rate and the loading curvature, which show that the average strain rate can express as the representative of strain rate for the indentation technique.

\section{Summary}

In order to clarify the effect of high strain rate on the indentation technique, the effect of strain rate distribution of the indentation on pure aluminum with respect to the strain rate dependence of strength was investigated. The obtained results are summarized as follows.

(1) The effect of strain rate on the indentation during the loading was replicated using the implicit method, as is the case in the explicit method.

(2) The strain rate distribution was quite different from the strain distribution, which showed that the strain rate at the crater rim was higher than that beneath the indenter.

(3) The strain rate distributions of the same indentation strain rate have the similarity rule.

(4) We established the calculation method of the average strain rate, which can express as the representative of strain rate for the indentation technique. 
This study was supported in part by the Grant-in-Aid for Young Scientists (A) (Grant No. 25709004) from the Ministry of Education, Culture, Sports, Science and Technology, Japan. The authors also wish to thank the Light Metal Educational Foundation, Inc., Osaka in Japan for financial support.

\section{References}

[1] Y.T. Cheng, C.M. Cheng, Mater. Sci. Eng. R44, 91 (2004).

[2] A. Gouldstone, N. Chollacoop, M. Dao, J. Li, A.M. Minor, Y.-L. Shen, Acta Mater. 55, 4015 (2007).

[3] N. Ogasawara, X. Chen, N. Chiba, J. Mater. Res. 21, 947 (2006).
[4] M.F. Doerner, W.D. Nix, J. Mater. Res. 1, 601 (1986).

[5] E. Harsono, S. Swaddiwudhipong, Z.S. Li, L. Shen, Int. J. Solids Structures 48, 972 (2011).

[6] H. Yamada, Y. Shimizu, N. Ogasawara, X. Chen, J. JSEM 12, 88 (2012).

[7] L. Liu, N. Ogasawara, N. Chiba, X. Chen, J. Mater. Res. 3, 784 (2009).

[8] N. Ogasawara, H. Yamada, Proc. JSEM 2010 Annu. Conf. Exp. Mech., 247 (2010).

[9] M. Dao, N. Chollacoop, K.J. Van Vliet, T.A. Venkatesh, S. Suresh, Acta mater. 49, 3899 (2001).

[10] N. Ogasawara, N. Chiba, X. Chen, J. Mater. Res. 20, 2225 (2005). 